\title{
The Application of Pricing Model in Self - Service Crowdsourcing Platform
}

\author{
Zhi-Peng MA ${ }^{1, a}$, Shu-Wan YAO ${ }^{1, b}$, Ke-Fei $X U^{1, c}$ and Ji-Li WANG ${ }^{1, d, *}$ \\ ${ }^{1}$ Jilin University, Changchun, China, 130012 \\ a1583244282@qq.com, b745841115@qq.com, '1047001337@qq.com, \\ dwangjili1100@126.com
}

Keywords: Crowdsourcing, Pricing rules, Incentive pricing model, Fitting with Matlab

\begin{abstract}
The crowdsourcing self-service model is an important part in new Internet business. In this case, setting the price is the core issue of the model. In order to provide a reasonable price and stimulate the enthusiasm of members of the website, this paper proposed a model of 'incentive pricing scheme' through analysis of a large amount of data. The price includes the basic amount and incentive reward amount in two parts. The basic amount is a function of four variables of the mission, including location, the membership location, the membership task quota and the mission density. Incentive reward amount is a function of the member's performance coefficient weighted by the coefficient of variation method. Matlab is utilized to fit data and get the coefficient. The pricing model can be used to analyze the real data set to get a reasonable task pricing scheme.
\end{abstract}

\section{Introduction}

'Making money by taking a photo' is a crowdsourcing self-service model in new business on the Internet. Firstly, users download the APP and register as a member of the APP. Then they can earn remuneration after finishing a task assigned by the App. Usually, the tasks are taking the photos needed to improve the map or so on. Crowdsourcing is the key service model.

The term "crowdsourcing" was first proposed by Jeff Howe[1] in Wired in the United States in 2006. Crowdsourcing refers to a company or organization in the past by the staff to perform the tasks of the work in a voluntary manner to outsource to the unspecified mass Network approach.

Dominic[2] pointed out that the crowdsourcing bidding behavior is similar to the reverse auction bidding, and has the characteristics of reverse and risk-profit. Using the game theory model to study the quantitative relationship between the bonus and the received works, Dominic found that awards have obvious consistency with the number of participants Logarithmic form of decreasing growth. Christian and $\mathrm{Yi} \mathrm{Xu}$ [3] designed multiple rounds of competition laws to reduce the loss caused by participants' sabotage. Nikolay[4][5] recommended to establish a member honor system. Ma Yan[6] designed the equilibrium pricing and cost gradient based scheduling strategy of scientific workflow to achieve maximal profits of service providers.

This crowdsourcing self-service platform on Internet[7] provides enterprises with various business inspections and information collection, which can save costs compared with the traditional market research methods and ensure the authenticity of investigation data and shorten the investigation cycle. Therefore, the APP becomes the core of platform operation. Moreover, making reasonable price for the tasks in APP is the crucial element. If the pricing is not appropriate, none of the members would like to accept the task, which led to the failure of commodity inspection.

In consideration of the statement above, this article analyzes the pricing problems in crowdsourcing model and proposes a model of 'incentive pricing scheme'.

\section{Research about the Rule of Pricing}

What is needed for a crowdsourcing model is a pricing method that is suitable for service products. The traditional pricing rules of service products are divided into cost-oriented pricing, 
demand-oriented pricing and competition-oriented pricing. [8]

Among them, homogeneous procedural service products often take cost-guided pricing method, which makes sure that the service business is fully compensated for the Consume of resources and get a certain profit. On the other hand, R \& D-based services tend to take competitive-oriented pricing method. That is, they set the price on the base of the competition in the market.

In the crowdsourcing model, the mature cost-oriented pricing method is most widely used. At the same time, the intense competition of crowdsourcing platform makes the task of publishers and receiving the task of membership tend to competitive-led pricing. Brabham[9] found that the main motivation of participants was others' recognition and bounty from the website. Therefore, this article will be integrated cost-oriented pricing and competition-oriented pricing method, put forward the relevant models.

This data used in this paper is from the related accessories of 2017 National Undergraduate Mathematical Contest Modeling B pricing the 'photo-making task ’.[10]

\section{Data Source.}

This paper analyzes 835 task points and 1877 member data, including the latitude and longitude of the task, the pricing of the task, the latitude and longitude of the member location, the scheduled task limit and the credit value. The total amount MW allocated to 835 task points is 57,707.5 yuan, with an average amount of 69.11 yuan.

\section{Program Development.}

The theory of motivation points out that either extrinsic motivation or internal motivation will, to a certain extent, affect one's behavior. [11]This paper uses a 'incentive pricing program', the main purpose is to raise the share of the higher pricing range, in order to motivate members with high credit to accept the task, but also to make most members benefit. Based on the conditions above, the price of each task $\mathrm{W}$ is divided into two parts, the basic amount $\mathrm{W} 1$ and incentive reward $\mathrm{W} 2$. The total amount of the two parts is called MW1 and MW2, MW = MW1 + MW2.

\section{Model Establishment}

\section{The Establishment of Model of Basic Amount Price.}

W1 is a function that has nothing to do with the member's creditworthiness. Inferred from the data collected, W1 is related to the four points including task position $\alpha$, member position $\beta$, member task limit $\gamma$ and task density $\rho 1$,

$$
W 1=f(\alpha, \beta, \gamma, \rho 1) \text {. }
$$

Thereinto, $\alpha=h 1(x, y), \beta=h 2(x, y), \mathrm{x}$ is the longitude coordinate and $\mathrm{y}$ is the latitude coordinate.

\section{The Relationship between Task Price and the Location of the Task.}

Firstly, the task price and location distribution are drawn based on the collected data, then the fuzzy clustering algorithm is used to aggregate the task points into three categories, as shown in Figure 1. 


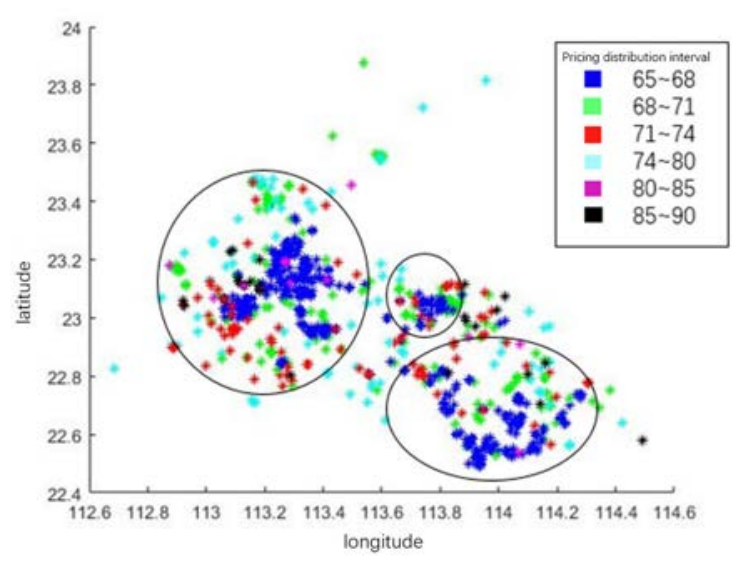

Figure. 1 Task Price Distribution

It can be drawn that there is a certain connection between price and the location of a task. The three oval areas in Figure 1 are centered on the cluster center, which is called the cluster of mission points.

Make the distance between the task point and the nearest cluster center $\mathrm{d}$. The task price will be proportional to $\mathrm{d}$.

\section{Relationship between Task Price and Member Location.}

There are 3 locations in the location map that are more closely related to the location of the mission, which is called the concentration area. The concentration of similar locations called similar concentration area.

It is assumed that the member position is a single-valued function of the mission position, that is

$$
\beta=g(\alpha) \text {. }
$$

Make the center of similar concentration of L, remember longitude X, latitude Y.

$$
L=\sqrt[p]{\sum_{k=1}^{n}\left|x_{1 k}-x_{2 k}\right|^{p}}
$$

Make $\mathrm{P}=1$, that is, use Manhattan distance to calculate the distance.

Calculated by Matlab, the center distances of three similar concentrated areas are respectively 0.0513 and 0.90370 .9275 , which satisfy the requirements of positive correlation.

\section{The Relationship between Task Price and Task Density.}

Define the number of tasks per unit area for the mission density $\rho 1$, the number of members per unit area membership density $\rho 2$. In each centralized area, the closer to the regional center, the higher the task density and membership density, the lower the task pricing, that is, the task pricing is negatively related to $\rho 1$ and $\rho 2$. That is, $\frac{\partial W 1}{\partial \rho_{1}}<0, \frac{\partial W 1}{\partial \rho_{2}}<0$. Besides W1 has upper and lower bounds. The following relationship is drawn:

$$
W 1 \propto\left[k_{0}+k_{1} \operatorname{arccot}\left(k_{2} \rho_{1} \rho_{2}+k_{3}\right)\right] .
$$

k0, k1, k2, k3 are coefficients, k0, k1 are positive.

\section{The Relationship between Task Pricing and Membership Task Quota.}

Through analysis, the task price and the task limit of members nearby is a positive correlation. In each concentration area, the closer to the regional center, the higher the task density and membership density, the lower the task limit, that is, the task quota and membership density are negatively 
correlated.

In summary, the formula of task price $\mathrm{W}$ is

$$
W=\left(a_{0} \alpha+a_{1} \beta\right) \gamma\left[k_{0}+k_{1} \operatorname{arccot}\left(k_{2} \rho_{1} \rho_{2}+k_{3}\right)\right] .
$$

$\mathrm{a} 0$, a1, a2 is the coefficient.

Concluded the statement above:

$$
W 1=\left[a_{0} h 1(x, y)+a_{1}(1-L) h 1(x, y)\right] \gamma\left[k_{0}+k_{1} \operatorname{arccot}\left(k_{2} \rho_{1} \rho_{2}+k_{3}\right)\right] .
$$

\section{The Establishment of Model of Incentive Reward Amount Price.}

Ria Mae Borromeo[12] pointed that Task assignment requires creativity and the gap should be widened. Dominic Dipalantino and Milan Vojnovic[13] assumed that if in the risk-neutral condition for the members, the likelihood of success of the tasks posted by the task publisher increases logarithmically as the amount of the reward increases. That is, as the amount of the reward increases to a certain extent, the impact of the increase of the amount on the success rate will be reduced.

In the program, the incentive reward amount is mainly affected by the indicators of the members, including member density $\rho 2$, membership task limit $\gamma$, membership credit $\omega$. Before the analysis, task quotas are partitioned and each grid is unit area, as shown in Figure 2.

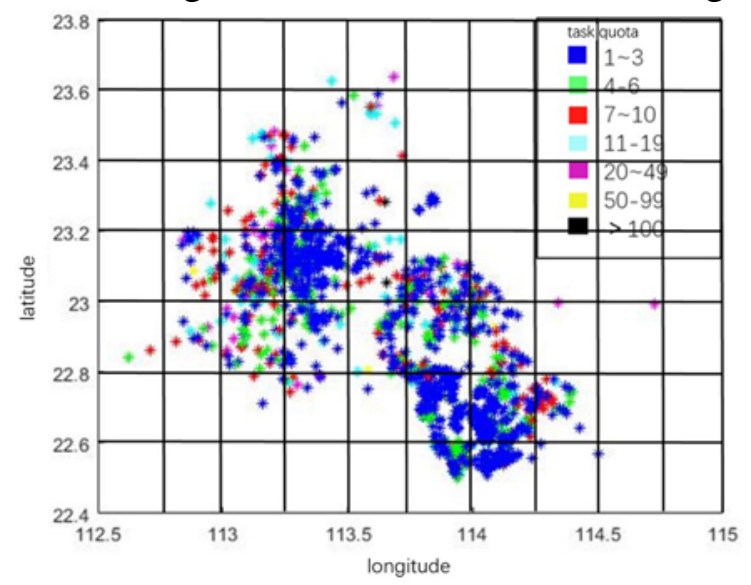

Figure. 2 Task Quota Partition Map

In order to make a more rational program, the concept of performance coefficient $\mathrm{v}$ is introduced.

For member i, performance coefficient equals task limit multiplied by credit.

$$
v_{i}=\gamma_{i} \times \omega_{i}
$$

Referring to Terwiesch's model of considering the uniqueness of product transactions [14], The coefficient $\mathrm{V}$ for each item in the region is

$$
V=\rho_{2} \max \left\{v_{i}\right\}+\left(\rho_{\max }-\rho_{2}\right) \frac{\sum_{i=1 \ldots n} v_{i}}{n} .
$$

Then to the right of the equation represents the population in the region. In the distribution of the reward amount, the weight of each member $\lambda i$ is determined by the use of coefficient of variation and normalization.

\section{Result of the Model.}

Based on the above analysis, the final result of this pricing plan is 
$W=W 1+W 2=W 1+\lambda_{i} M W 2$.

The formulas for $\mathrm{W} 1$ and $\lambda \mathrm{i}$ are shown in Eqs. (6) and (8).

\section{Model Application.}

The collected data is applied to the established model for further analysis.

The total amount in the Data is 57,707.5 yuan. According to the model, $70 \%$ of the amount is allocate to be the basic amount MW1, and 30\% of the amount is allocate to be incentive reward MW2. Namely MW1 is 40395.3 yuan, MW2 is 17312.2 yuan.

In order to simplify the calculation, the number of tasks is divided into grids which involve 0.2 longitude and 0.2 dimensions. Each divided grid is called a unit area. As a result, the distribution of tasks is shown in Fig. 3 and Fig. 4.

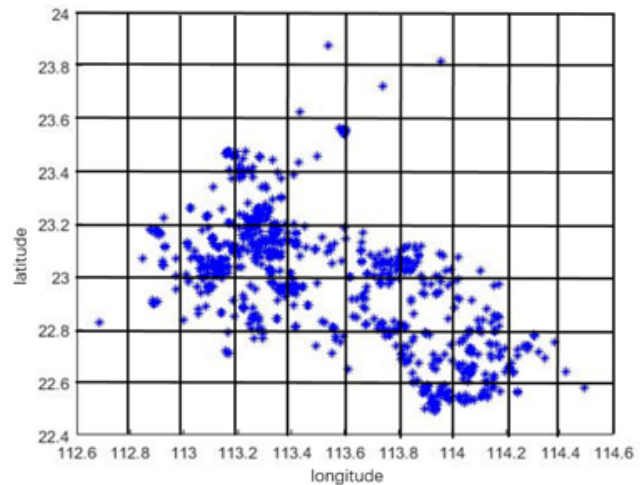

Figure. 3 Density of Task Grid Diagram

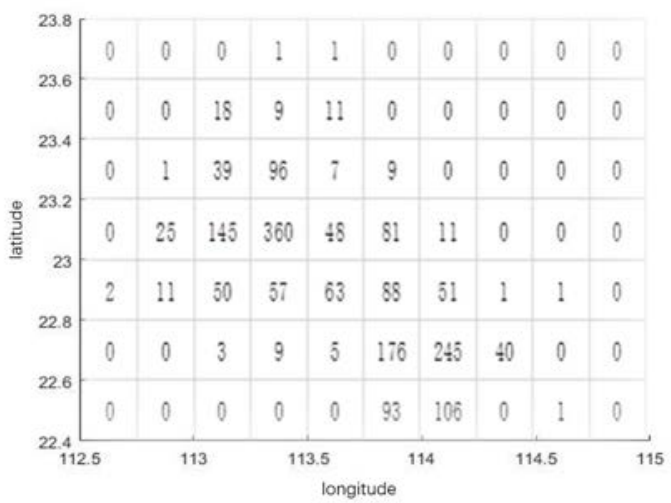

Figure. 4 the Density of each Grid

The coordinates of each grid center point are the coordinate of the mission point (x0, y0) in the grid. Make $^{h 1}(x, y)=\sqrt{x^{2}+y^{2}}$. The number of tasks per unit area is taken as the value of $\rho 1$, and the number of arithmetic average tasks per unit area is taken as $\gamma$. These two values are the parameters in Equation (6.)

Use MATLAB to fit, the coefficient matrix can be concluded.

$$
\left[\begin{array}{l}
k_{0} \\
k_{1} \\
k_{2} \\
k_{3} \\
a_{0} \\
a_{1}
\end{array}\right]=\left[\begin{array}{l}
36.07 \\
-2.22 \\
0.05 \\
-0.47 \\
2.18 \\
3.07
\end{array}\right] .
$$

After normalizing W1 and W2, the latest pricing can be figured out. The table 1 and table 2 shows the latest price of the first 20 task and the last 20 tasks. 
Table 1 the price of Top 20 tasks

\begin{tabular}{|c|c|c|c|}
\hline Task number & $\begin{array}{c}\text { Latest } \\
\text { price/RMB } \\
\text { yuan }\end{array}$ & $\begin{array}{c}\text { Task } \\
\text { number }\end{array}$ & $\begin{array}{c}\text { Latest } \\
\text { price/RMB } \\
\text { yuan }\end{array}$ \\
\hline A0798 & 227.2222 & $\mathrm{~A} 0775$ & 762.048 \\
\hline A0797 & 265.0959 & $\mathrm{~A} 0773$ & 838.7026 \\
\hline A0796 & 359.9914 & $\mathrm{~A} 0765$ & 1048.731 \\
\hline $\mathrm{A} 0794$ & 378.333 & $\mathrm{~A} 0760$ & 1104.777 \\
\hline $\mathrm{A} 0793$ & 399.5956 & $\mathrm{~A} 0751$ & 1199.243 \\
\hline $\mathrm{A} 0791$ & 409.3573 & $\mathrm{~A} 0750$ & 1617.378 \\
\hline $\mathrm{A} 0790$ & 499.4741 & $\mathrm{~A} 0749$ & 1730.029 \\
\hline $\mathrm{A} 0789$ & 560.2161 & $\mathrm{~A} 0742$ & 1793.802 \\
\hline $\mathrm{A} 0788$ & 650.1481 & $\mathrm{~A} 0739$ & 2810.414 \\
\hline
\end{tabular}

Table 2 the price of the last 20 tasks

\begin{tabular}{|c|c|c|c|}
\hline Task number & $\begin{array}{c}\text { Latest } \\
\text { price/RMB } \\
\text { yuan }\end{array}$ & Task number & $\begin{array}{c}\text { Latest } \\
\text { price/RMB } \\
\text { yuan }\end{array}$ \\
\hline $\mathrm{A} 0284$ & 32.55677 & $\mathrm{~A} 0752$ & 32.57096 \\
\hline $\mathrm{A} 0289$ & 32.55677 & $\mathrm{~A} 0253$ & 32.57124 \\
\hline $\mathrm{A} 0291$ & 32.55677 & $\mathrm{~A} 0248$ & 32.58516 \\
\hline $\mathrm{A} 0293$ & 32.55677 & $\mathrm{~A} 0220$ & 32.58941 \\
\hline $\mathrm{A} 0255$ & 32.57096 & $\mathrm{~A} 0239$ & 32.58941 \\
\hline $\mathrm{A} 0257$ & 32.57096 & $\mathrm{~A} 0134$ & 32.59935 \\
\hline $\mathrm{A} 0262$ & 32.57096 & $\mathrm{~A} 0142$ & 32.59935 \\
\hline $\mathrm{A} 0263$ & 32.57096 & $\mathrm{~A} 0144$ & 32.59935 \\
\hline $\mathrm{A} 0276$ & 32.57096 & $\mathrm{~A} 0145$ & 32.59935 \\
\hline
\end{tabular}

Compared with the original plan, the standard deviation of the price of the new plan is 293.8, and the distribution is more discrete. In the case of a certain cost, the disparity between high-priced tasks and low-cost tasks increases. So the incentive effect is higher than the original program. At the same time, the tasks are distributed in the form of task packages, which fully mobilize the enthusiasm of members in the region and at the same time alleviate the competition among members so as to improve the success rate of tasks.

\section{Conclusion}

The paper proposes a model of 'incentive pricing scheme' through analysis of a large amount of data, including the basic amount and incentive reward amount. The advantage of the model is that the relationship between the pricing and each variable is expressed by the function, which is clear and concise. At the same time, the model introduces the concept of 'coefficient of performance' innovatively, and encourages members to participate through the distribution of reward, so as to improve the completion rate. The disadvantage is that the fitting efficiency of the model needs to be improved.

All in all, the crowdsourcing model can significantly reduce business manpower costs and improve efficiency. This emerging business model is promising in the future. Further, optimizing algorithms of task pricing can greatly enhance the public's participation in the crowdsourcing project. Crowdsourcing implementations are seeking diverse and creative contributions and employing optimization with a richer set of mechanics [15]. Therefore, the issue of task pricing deserved to be researched more deeply. 


\section{References}

[1]HOWE J. The rise of crowdsourcing [J].Wired, 2006, 14(6):176-183.

[2]Dominic Dipalantino.2009. Milan Vojnovic. Crowdsourcing and All-Pay Auctions [J]. Operations Research Letters, 10: 119-128.

[3]Christian Terwiesch. Yi Xu. 2008. Innovation Contests. Open Innovation, and Multiagent Problem Solving [J]. Management Science, 2008.9(54): 1529-1543.

[4]Nikolay Archak. Money, Glory and Cheap Talk: Analyzing Strategic behavior of Contestants in Simultaneous Crowdsourcing Contests on Top Coder.com [J]. International World Wide Web Conference Committee, 2010.4: 1-10.

[5]Nikolay Archak. Arun Sundararajan. Optimal Design of Crowdsourcing contests [J]. International Conference on Information Systems, 2009.1-19.

[6]Ma Yan, Gong Bin, Zou Lida. Equilibrium Pricing and Cost Gradient Based Scheduling Strategy of Scientific Workflow [J]. ACTA ELECTRONICA SINICA, 2010.10(10):2416-2421.

[7]Araz Taeihagh. Crowdsourcing: a new tool for policy-making? [J]. Policy Sci (2017) 50:629-647.

[8]Liu Xiaogang. Factors Affecting Task Publisher to Bid in Crowdsourcing: An Empirical Study from China [D]. Chongqing: Chongqing University, 2012:18-20.

[9]Brabham. D. C. Crowdsourcing as a Model for Problem Solving [J]. The International Journal of Research into New Media Technologies, 2008.14(1): 75-90.

[10]http://www.mcm.edu.cn/html_cn/node/460baf68ab0ed0e1e557a0c79b1c4648.html

[11]Zhang Jun, Li Peng. Competition Network of Crowdsourcing Participators-Taking Zhubajie.com Website as an Example[J]. Journal of Intelligence, 2004.11:188-192.

[12]Ria Mae Borromeo, Thomas Laurent, Motomichi Toyama, Maha Alsayasneh, Sihem Amer-Yahia, Vincent Leroy. Deployment strategies for crowdsourcing text creation [J]. Information Systems, 2017(71):103-110.

[13]Dominic Dipalantino, Milan Vojnovic. Crowdsourcing and All-Pay Auctions [J]. Operations Research Letters, 10: 119-128.

[14]Terwiesch. C. Xu. Y. Innovation contests. open innovation, and multiagent problem solving[J]. Management Science, 2008.54(9): 1529-1543.

[15] Benedikt Morschheuser, Juho Hamari, Jonna Koivisto, Alexander Maedche. Gamified crowdsourcing: Conceptualization, literature review, and future agenda [J]. International Journal of Human-Computer Studies, 2017(106): 26-43. 